\title{
Moulting in the chalimus larva of the salmon louse Lepeophtheirus salmonis (Copepoda, Caligidae)
}

\author{
J.E. Bron, A.P. Shinn \& C. Sommerville \\ Institute of Aquaculture, University of Stirling, Stirling FK9 4LA, Scotland
}

Keywords: Lepeophtheirus salmonis, chalimus, Copepoda, moult, cuticle, ultrastructure, parasite

\begin{abstract}
The ultrastructure of moulting in the chalimus phase of the parasitic caligid copepod Lepeophtheirus salmonis is described for the first time. The major features of the moult sequence correspond to those of other Crustacea, save for the absence of a distinguishable intermoult as defined by reduced epidermal activity and a cessation of cuticle production. This latter may be explained either in terms of a continuous moulting process related to the constant food supply available to a parasite or in terms of a highly abbreviated intermoult period. The findings are discussed with respect to studies of other Crustacean species.
\end{abstract}

\section{Contents}

Introduction

Materials and methods

Results

Discussion

Summary

Acknowledgements

References

\section{Introduction}

Sea lice, in common with other crustacean groups studied, undergo periodic moults allowing changes in size and morphology between stages. Periodic moulting is necessary to all arthropods during growth phases given the constrictions of a hard exoskeleton. In other crustacean groups, and particularly in decapods, the moult cycle has been subdivided by many authors into a number of stages which rely for their recognition upon morphological criteria assessed by physical attributes (e.g. hardness of the cuticle) and appearance as noted by naked eye, light microscopic or electron microscopic observation (inter alia Drach, 1939; Drach \& Tchernigovtzeff, 1967; Skinner 1962, 1985). According to the stages recognised by Skinner (1962), following shedding of the old cuticle during ecdysis (Stage E) comes metecdysis or postmoult (Stages $A-\mathrm{C}_{3}$ ) during which the new endocuticle (inner procuticle) is completed, anecdysis or intermoult (Stage $\mathrm{C}_{4}$ ) a resting phase which follows completion of the cuticle and which may last for a considerable period in some species and finally, proecdysis or premoult during which time the old cuticle separates from the epidermis (apolysis) and is resorbed and finally shed as the new cuticle is constructed beneath it (stages $D_{0}$ $\mathrm{D}_{4}$ ). Subsequent authors who have tried to apply the proposed criteria to smaller species such as Daphnia magna Straus (Halcrow, 1976) have used electron microscope studies to establish similar stages since larger scale observations are not easily attainable.

Observations of moulting in copepods are rare. Only Dexter (1981) has made any attempt to tie moulting in a copepod (Calanus marshallae Frost) to those recognised by other authors and even in this case, only three stages - premoult, postmoult and intermoult were designated (according to observation of setogenesis using light microscopy). Observation of moulting in parasitic copepods is similarly sparse, with naked eye observations of moulting in mobile stages of Lepeophtheirus pectoralis (Müller) being provided by Anstensrud (1990) and scanning and transmission electron 
microscope studies of cuticle folding in moulting females by Smith \& Whitfield (1988) for Lernaeocera branchialis (L.).

The purpose of the work presented here is to describe the moult cycle in the chalimus phase of $L$. salmonis in order to provide a baseline for studies seeking to examine changes in cuticle structure / moulting which may arise as a result of exposure to exogenous compounds or from use of vaccines. An important facet of the present study was to tie observations, where possible, to the stages classically recognised in other crustacean groups.

\section{Materials and methods}

Gravid adult female sea lice were removed from farmed Atlantic salmon and larvae were hatched and reared in the laboratory at $10^{\circ} \mathrm{C}$. Infective copepodids were used to infect 20 Atlantic salmon. Single fish were sacrificed every $24 \mathrm{~h}$ and all attached larval stages removed, perforated to allow fixative penetration and fixed for transmission electron microscopy according to the methods of Eisenman \& Alfert (1982) modified by omission of the pre-fixation step. Chalimus II larvae, present from day 10 to day 18 post-infection, and thereby providing the longest larval moulting sequence for examination, were chosen as the subject for this study.

Specimens for TEM were post-fixed in $1 \% \mathrm{OsO}_{4}$, dehydrated through a graded acetone series and embedded in Spurr resin. The resin was polymerised at $70^{\circ} \mathrm{C} .80 \mathrm{~nm}$ sections were cut on a Reichert Ultracut $\mathrm{E}$ and stained with uranyl acetate and lead citrate according to the methods of Hayat (1989) and Reynolds (1963) respectively. The uranyl acetate method was modified by the use of methanol rather than water as the solvent. Grids were observed using a Philips 301 TEM running at $80 \mathrm{KV}$. Observations, except where stated, concern the dorsal cephalothoracic cuticle.

\section{Results}

The ultrastructure of the completed cuticle is given in the accompanying paper (Bron, Shinn \& Sommerville, this volume). The size of the chalimus II prevents the use of cuticle rigidity / appearance under light microscopy as a criterion for assessing the classical moult stages of Drach Tchernigovtzeff (1967). The aim of this work has therefore been to try to associate electron microscope observations made here with those of other authors working on species where such ultrastructural changes may be more formally linked to visual observations. Because of the short duration of the moult cycle and the variability of individual developmental progress seen, no attempt could be made to apply a time course to the morphological events described.

The moult cycle involves the entire cuticle inclusive of the buccal, foregut and hindgut cuticles. The newly moulted individual is recognisably paler than older animals, which is suggestive of subsequent cuticular tanning. At moult stage $A / B$ ("metecdysis"), following the moult from the chalimus I stage (moult stage $\mathrm{E}$ ), the procuticle in most areas comprises a reduced number of laminae although the epicuticle is complete. The apical membrane of the epidermis is not pronounced and can not easily be distinguished as a distinct entity due to a wide electron-dense band at the interface between the epidermis and the cuticle (Fig. 1a). This dense band is likely to be associated with deposition of further procuticle following the moult and represents a zone of disorganised cuticular components.

From this point onwards, i.e. during the further metecdysial stages B-C, the procuticle is elaborated upon in terms of the addition of further laminae although these are generally not morphologically distinguishable into $\mathrm{p}^{1}$ and $\mathrm{p}^{2}$. In most areas the new laminae, whilst usually barely or not at all distinguishable from those laid down before the preceding moult, may sometimes be closer together

Key to abbreviations used in figures: A Apical membrane; E Epicuticle; EC Exuvial Cleft; Ep Epidermis; Op Old procuticle; $\mathbf{P}$ Procuticle, Tf Tonofilament; tpl Transitional procuticle, V Vesícle.

Fig. Ia. Electron micrograph of metecdysial (Stage A/B) cuticle showing completed epicuticle and small number of procuticular laminae. Note the electron dense band overlying the epidermis which is thought to represent disorganised procuticular components in process of production. Scale bar $=0.2 \mu \mathrm{m}$ 


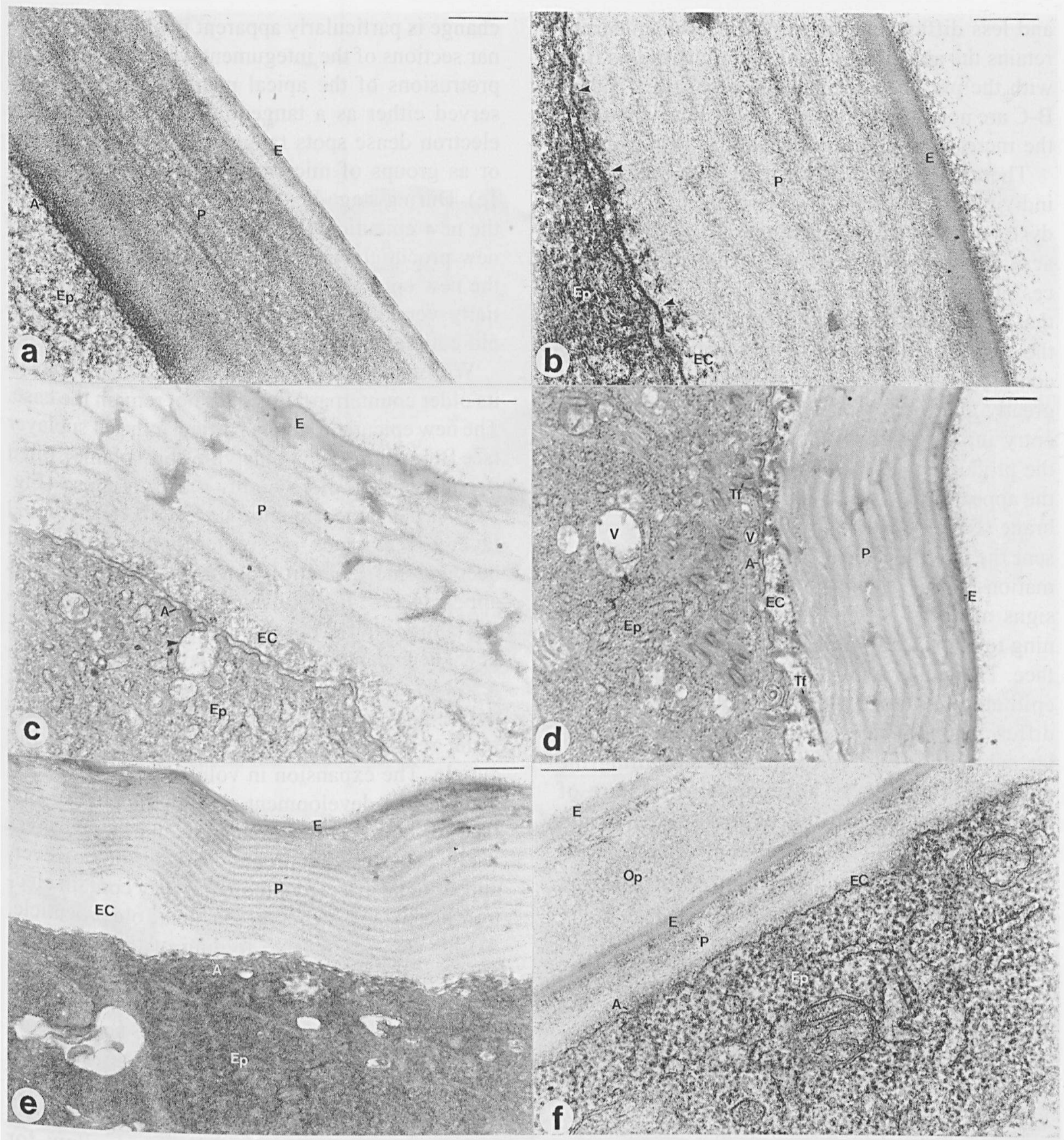

Fig. $1 \mathrm{~b}$. Electron micrograph of early pröecedysial cuticle (Stage $\mathrm{D}_{0}-\mathrm{D}_{1}$ ) showing presence of ecdysial membrane (arrowed) and the development of an exuvial cleft as apolysis proceeds. Scale bar $=0.2 \mu \mathrm{m}$

Fig. Ic. Electron micrograph of early proecdysial cuticle (Stage $D_{0}-D_{1}$ ) showing development of exuvial cleft with fibrillar contents. A secretory vesicle (arrowed) may be observed fusing to the apical membrane. Scale bar $=0.5 \mu \mathrm{m}$

Fig. Id. Electron micrograph of early proecdysial cuticle (Stage $\left.\mathrm{D}_{0}-\mathrm{D}_{1}\right)$ showing tonofibrils passing across cleft to insert on the old cuticle. Also apparent are numerous vesicles within the epidermal cytoplasm and in the vicinity of the apical membrane. Scale bar $=$
$0.5 \mu \mathrm{m}$

Fig. le. Electron micrograph of early proecdysial cuticle (Stage $\mathrm{D}_{0}-\mathrm{D}_{1}$ ) showing microvillous surface of apical membrane (apparent Fig. If E Electron dense foci representing transversely sectioned microvilli). Scale bar $=0.5 \mu \mathrm{m}$

is alread Electron micrograph of proecdysial cuticle (Stage $\mathrm{D}_{2}$ late) showing early stages of procuticle construction. The new epicuticle is already present and abuts the base of the old cuticle. Scale bar $=0.5 \mu \mathrm{m}$ 
and less diffuse in their structure. The epidermis retains the appearance of high activity at this time with the presence of abundant organelles. Stages B-C are not morphologically recognisable save for the increase in numbers of laminae displayed.

There has been no indication within the many individuals examined of a true stage $\mathrm{C}_{4}$ ("anecdysis") in that there is no appearance of reduced activity within the epidermis that might signal cessation of cuticulogenesis. This suggests either that within the chalimus II larva, preparation for the next moult proceeds immediately following completion of the cuticle, or that this stage has a greatly curtailed duration. The first indication of entry into "proecdysis" (putative stage $D_{0}-D_{1}$ ) is the production by the epidermis of a layer having the appearance of a second apical (ecdysial) membrane (Fig. 1b) although this structure may represent the precursor of the new epicuticle. The formation of this layer is accompanied by the first signs of apolysis, with the old procuticle beginning to come away from the apical epidermal surface. The gap or exuvial cleft formed between the epithelium and the old procuticle is filled with diffuse material having the appearance of a fibrillar substance (Fig. 1c). The material filling the exuvial cleft is likely to represent a mixture of products including not only enzymes secreted into the cleft from the epidermis but also material resulting from the dissolution of the old cuticle. In some sections, vesicles can be seen fusing to the apical membrane which probably represent secretion of material into the cleft (Fig. 1c). Tonofibrils may be clearly seen crossing the exuvial cleft to their points of insertion in the old cuticle, although there is no indication that tonofibrils continue to insert in the old procuticle once the new procuticle has been laid down (Fig. 1d).

As the exuvial cleft widens, the apical membrane of the epidermis becomes more rugose. This change is particularly apparent in oblique and planar sections of the integument where microvillous protrusions of the apical membrane may be observed either as a tangentially cut line of more electron dense spots ranged along the membrane or as groups of microvilli cut transversely (Fig. 1e). During stage $D_{2}$, following the completion of the new epicuticle in early $\mathrm{D}_{2}$, the first laminae of new procuticle are also laid down (late $\mathrm{D}_{2}$ ). Both the new epicuticle and the new procuticle are initially very similar in appearance to those of the old cuticle (Fig. 1f).

Whilst the new epicuticle is initially similar to its older counterpart, this does not remain the case. The new epicuticle, and in particular the e4 sublayer (see Bron, Shinn \& Sommerville, this volume), often becomes much more highly electron dense (Fig. 2a). At the same time, the underlying transitional layer tp1 expands in volume considerably and becomes more electron dense and granular in its appearance - distinguishing it from the underlying laminae of the developing procuticle (Figs. $2 b$, 2c). The expansion of the tpl layer is probably the result of material passing through from the epidermis and, more importantly, absorption of material resulting from the dismantling of the old cuticle. The expansion in volume of the tp1 layer leads to the development of a highly rugose surface for both the new epicuticle and the tp1 layer itself. The subjacent procuticle does not, however, appear to be affected although it may contain electron-lucent lacunae not present in the old procuticle. At this stage, extensive folding of the epidermal apical membrane is frequently apparent.

During the laying down of the new cuticle, large folds (macro-folds) of new cuticle secreted by epidermal invaginations also become apparent beneath the old cuticle (Figs. $2 \mathrm{~d}, 2 \mathrm{e}$ ). The scale of these folds is greater than the depth of the old cuticle and their appearance is presumed to allow for expansion of the larval volume following ecdysis.

Key to abbreviations used in figures: A Apical membrane; E Epicuticle; EC Exuvial Cleft; Ep Epidermis; Op Old procuticle; $\mathbf{P}$ Procuticle, Tf Tonofilament; tp1 Transitional procuticle, V Vesicle.

Fig. 2a. Electron micrograph of proecdysial cuticle (Stage $\mathrm{D}_{2}$ late- $\mathrm{D}_{3}$ ) demonstrating highly electron dense epicuticle and expanded tpl layer in developing cuticle. Tonofibrils (arrowed) visible within the old procuticle are no longer connected to the epidermis. Scale bar $=0.5 \mu \mathrm{m}$

Fig. 2b. Hïgher magnification electron micrograph of proecdysial cuticle (Stage $\mathrm{D}_{2}$ late- $\mathrm{D}_{3}$ ) showing expanded tpl layer and rugose nature of tp1 and epicuticle. The observed rugosity does not extend to the remaining procuticle. The apical membrane is also highly invaginated (arrowed). Scale bar $=2 \mu \mathrm{m}$ 


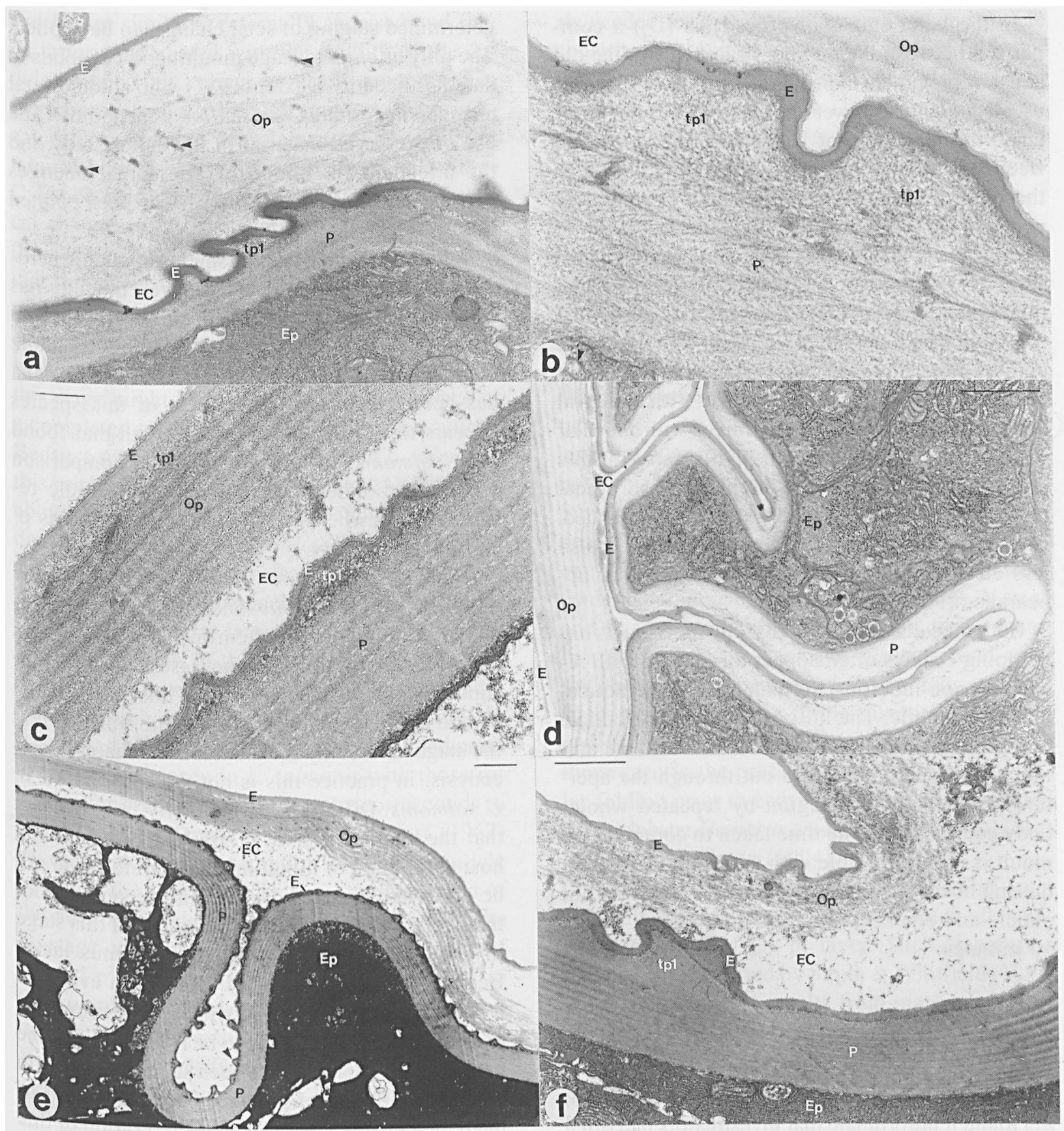

Fig. $2 c$. Electron micrograph of proecdysial cuticle (Stage $\mathrm{D}_{2}$ late- $\left.\mathrm{D}_{3}\right)$ illustrating difference between epicuticle and tpl layers of old and developing cuticles. Numerous electron lucent inclusions are present within the developing procuticle but absent within that of the old procuticle. Scale bar $=0.25 \mu \mathrm{m}$

Fig. 2d. Electron micrograph of proecdysial cuticle (Stage $\mathrm{D}_{2}$ late- $\mathrm{D}_{3}$ ) demonstrating scale of macrofolding which includes the whole cuticular depth. Note the highly active epidermis underlying the developing cuticle. Scale bar $=1 \mu \mathrm{m}$

Fig. $2 e$. Electron micrograph of late proecdysial cuticle $\left(\right.$ Stage $\left.\mathrm{D}_{3}-\mathrm{D}_{4}\right)$ demonstrating the contrast between macrofolding of the whole cuticular depth and microfolding (arrowed) of the epicuticle and underlying tpl layer. Note the degenerate nature of the old cuticle. Scale bar $=1 \mu \mathrm{m}$

Fig. 2f. Electron micrograph of late proecdysial cuticle (Stage $\mathrm{D}_{4}$ ) prior to ecdysis (Stage E). Degenerate old cuticle shows little structure and is barely attached to the developing cuticle. Note the increasingly homogeneous nature of the tpl layer of the developing cuticle and continuing high activity of the epidermis. Scale bar $=1 \mu \mathrm{m}$ 
In the latter stages of proecdysis $\left(D_{4}\right)$ a comparison between the number of laminae in the old and new procuticle indicates that the new procuticle usually comprises fewer laminae than the old. Similarly, the laminae that have been laid down are frequently deeper and more diffuse than those of the preceding cuticle. Approaching the point of ecdysis (Stage E), the old cuticle has lost much of its structure, with only the epicuticle appearing intact (Fig. 2f). Within the old procuticle the fibres of the laminae appear very disorganised and are no longer bound together in coherent lamellae. The exuvial cleft has widened substantially as the old cuticle comes away and is filled with material having a fibrillar appearance admixed with cuticular debris. The epidermis is still highly active at this point and still displays a highly rugose apical membrane. The new tp1 layer, whilst still expanded, is less heterogeneous in its appearance and also less electron dense, having a finely granular appearance (Fig. 2f).

Under the dissecting microscope individuals undergoing ecdysis often appear misshapen with respect to non-moulting individuals and are noticeably less mobile. The initial rupture of the cuticle occurs in the chalimus cephalothorax and the animal subsequently squeezes out through the aperture produced in this region by repeated wholebody contractions. The time taken to complete the moult is highly variable with some individuals requiring hours to fully divest themselves of the old cuticle and others completing the exercise within 10 minutes.

\section{Discussion}

With regard to moult staging, Stevenson (1985: 21) found it unfortunate that investigators had often attempted to establish "their own arbitrary criteria for each group studied" rather than using criteria suggested by Drach $(1939,1944)$, Drach \& Tchernigovtzeff (1967) and Stevenson (1968). Whilst these suggested criteria are appropriate for the larger species to which they have been applied, development of refined techniques has allowed study of moulting in animals that are too small or frail for accurate estimates of cuticle hardening or visually determined staging of setal changes to be applied. The only attempt to stage moulting in copepods to date has been by Dexter (1981) who studied setal changes in Calanus marshallae copepodids. The use of preserved material in this latter study and the attendant likelihood of artefactual changes particularly relating to shrinkage has been pointed out by Buchholz (1982).

Without recourse to assessments of cuticle hardness and setal development the present study has sought to tie changes seen to those recorded in other species. In particular the study by Buchholz \& Buchholz (1989) on Euphausia superba Dana has been used, since the cuticle of this species appears to be most closely allied with that found in $L$. salmonis. In order to facilitate comparison with other studies, the exo- and endocuticle referred to elsewhere will here be referred to as $\mathrm{p}^{1}$ and $\mathrm{p}^{2}$ respectively.

Moult stages A-C are very difficult to determine precisely in $L$ salmonis since the criteria generally used to determine them in the other species have relied principally upon assessing cuticular rigidity and setal development. Whilst the $A_{1}$ stage should theoretically be easily diagnosable, as it is the stage entered by the animal immediately postecdysis, in practice this is not straightforward in L. salmonis. Difficulties arise firstly from the fact that the chalimus larva is attached to its salmon host at the time of moulting and therefore can not be observed to moult directly and secondly from the likelihood of a very short duration of this stage in the rapid moult cycle of the chalimus larva. Because sampling was only undertaken every 24 $\mathrm{h}$ it is likely that this stage will have been missed and was therefore unlikely to be represented in the present study. Stage $A_{2}$ has been suggested to begin with the initiation of post-ecdysial production of the $\mathrm{p}^{2}$ layer (Stevenson, 1968). Observation of this event classically requires recognition of external changes, which is again impossible under the normal conditions of chalimus development, although recognition of production of the $\mathrm{p}^{2}$ ultrastructurally should be possible where the $\mathrm{p}^{2}$ layer may be differentiated from the $\mathrm{p}^{1}$ layer. Stevenson (1968) also expressed concerns over the possibility for recognition of such a stage in cases where cuticle production did not stop during ecdysis. Stages $B_{1}$ 
and $B_{2}$ are similarly externally assessed and rely on criteria associated with the rigidity of the cuticle. Again, this cannot be easily recognised in the present species, although stages $\mathrm{A} / \mathrm{B}$ are indicated by a reduced complement of procuticular laminae with respect to the completed cuticle. Stage $C_{1}$ has been proposed to be reached when the chemical changes in the $\mathrm{p}^{2}$ layer have been completed (Stevenson, 1968) and again, it has been suggested that this event be linked where possible to externally recognisable events. Stage $\mathrm{C}_{4}$ comprises completion of the cuticle and hence the initiation of the "intermoult" phase of the cycle. In Orconectes this stage might be recognised by the presence of a completed membranous layer within the cuticle. Because such a layer does not occur in $L$. salmonis, this criterion clearly can not be used. As indicated above there is, furthermore, no indication of reduced epidermal activity in $L$. salmonis that might suggest true anecdysis. Instead, this stage can only be recognised by attainment of the full complement of procuticular laminae.

The separation of the old cuticle from the epidermis, "apolysis", is well defined in this study. Because such a change is universal and clearly recognised ultrastructurally, it presents a good point to tie the cycle seen in $L$. salmonis with those recorded for other groups. This stage corresponds to stage $D_{0}-D_{1}$ of Drach \& Tchernigovtzeff (1967) according to whom, secretion of new epicuticle begins at the start of the $D_{2}$ stage and secretion of the new procuticle $\left(p^{1}\right)$ towards the end of it. The $D_{3}$ stage corresponds to the resorption of most of the old $p^{2}$ layer and the $D_{4}$ stage corresponds to the completion of resorption of the $\mathrm{p}^{2}$ layer and the splitting of the exuvium prior to moulting. Stage E correspond to ecdysis of the exuvium.

In contrast to most studies of crustacean moult cycles, the epidermis of $L$. salmonis did not display a clear cycle of structural organisation or cellular activity such as has been described by Green $\&$ Neff (1972) for Uca pugnax (S.I.Smith) and instead gave indications of continuous activity with no clear intermoult period $\left(\mathrm{C}_{4}\right)$ being displayed. This may result from the rapid transition between moults and from the fact that there is a constant food supply available from the host which obviates the need for gradual accumulation of materi- als needed for growth, metamorphosis and cuticle construction. A similar lack of decline in structure or activity of the epidermis was also noted in Daphnia magna by Halcrow (1976) and in Euphausia superba by Buchholz \& Buchholz (1989) and in the former paper this was also suggested to result from a rapid transition time.

As opposed to the findings of Buchholz \& Buchholz (1989), there was no evidence in the present study of the extensive pleating of the apical plasma membrane although some signs of small microvilli were observed. There was, however, considerable evidence of large-scale folding (macro-folding) of the new cuticle which is suggested to allow expansion of the cuticle following ecdysis. Extensive cuticular folding of the trunk cuticle has been noted by Smith \& Whitfield (1988) for moulting females of Lernaeocera branchialis where it appeared to be restricted to the $p_{1}$ and epicuticle layers. This folding was suggested to result from epidermal folding in the moulting chalimus IV stage and was said to facilitate elongation during metamorphosis of fertilised females. Larger second-order folds were also described in L. branchialis and were suggested to perform a similar function. Such second-order folds appear closer in morphology to those seen in the present study. Extensive cuticular folding prior to ecdysis has also been reported for the lobster Homarus americanus Milne-Edwards by Cheng \& Chang (1994).

In the present study the new epicuticle / tp1 layer was demonstrated to be elaborated even after initial deposition of the $\mathrm{p}^{1}$ layer. Such elaboration has also been reported by Smith \& Whitfield (1988) although whether it results from material passing from the epidermis via the $p^{1}$ layer or through the addition of materials resulting from resorption of the old cuticle is unclear. The rugose surface of the new epicuticle / tpl layer may be allied to the first order folding of Smith \& Whitfield (1988) although it lacks the regularity of the folds demonstrated in that study and so may not be concerned with expansion of cuticle surface area following ecdysis.

As with the completed cuticle described elsewhere in this volume, there was no sign of intracuticular pore canals in the present study. Whilst these were suggested to be important for transport 
of material from the epidermis into the cuticle and for transport of material resorbed from the old cuticle in E. superba (Buchholz \& Buchholz 1989), it seems likely that the restricted cuticle thickness of $L$ salmonis chalimus larvae allows movement of material without such elaborations. Whilst Buchholz \& Buchholz describe the continued attachment of tonofibrils to the old cuticle despite the intercalation of the new $p^{1}$ and epicuticle and suggest that this may facilitate continued swimming in premoult euphausids, it seems likely that chalimus larvae are relatively inactive prior to the moult and therefore such an attachment is not necessary.

\section{Summary}

This study describes for the first time the ultrastructure of moulting in a copepod. The moulting sequence in the chalimus II larva of $L$. salmonis corresponds well to that of other crustacea described to date. In common with euphausids, no period of reduced activity was observed in the epidermis suggesting either that a defined intermoult is absent or that it is of very short duration. It is hoped that this description will provide a baseline for studies of the control of moulting in sea lice and of the effects of exogenous compounds on moulting.

\section{Acknowledgements}

The authors wish to acknowledge the assistance provided by all of the salmon farms that were visited in the course of these studies. The help provided by Maureen Menzies and Marguerite Kobs was also critical to the completion of this work. Funding for this work was provided by MAFF CSG.

\section{References}

Anstensrud M. 1990. Moulting and mating in Lepeophtheirus pectoralis (Copepoda: Caligidae), J. Mar. Biol. Ass. U.K. 70(2): 269-281.

Bron JE, Shinn AP, Sommerville, C. 1999. Ultrastructure of the cuticle in the chalimus larva of the salmon louse Lepeophtheirus salmonis (Krøyer, 1837) (Copepoda: Caligidae). This volume.

Buchholz C, Buchholz F. 1989. Ultrastructure of the integument of a pelagic crustacean: Moult cycle related studies on the Antarctic krill Euphausia superba. Mar. Biol. 101(3); 355-365.

Buchholz F. 1982. Drach's molt staging system adapted for Euphausiids. Mar, Biol. 66: 301-305.

Cheng J-H, Chang ES. 1994. Determinants of postmoult size in the American lobster (Homarus americanus). II. Folding of premoult cuticle. Can. J. Fish. Aquat. Sci. 51: 1774-1779.

Dexter BL. 1981. Setogenesis and molting in planktonic crustaceans. J. Plankton Res. 3: 1-13.

Drach P. 1939. Mue et cycle d'intermue chez les Crustacếs Décapodes. Amm. Inst. Oceanogr. 19: 103-391.

Drach P. 1944. Etude prèliminaire sur le cycle d'intermue et son conditionnement hormonal chez Leander serratus (Pennant), Bull. Biol. Fr. Belg. 78: 40-62.

Drach $P$, Tehernigovtzeff $C$. 1967. Sur la méthode de détermination des stades d'intermue et son application gènérale aux crustacếs. Vie Milieu 18: 595-607.

Eisenman EA, Alfert M. 1982. A new fixation procedure for preserving the ultrastructure of marine invertebrate tissues. J. Microsc. 125(1): 117-120.

Green JP, Neff MR. 1972. A survey of the fine structure of the integument of the fiddler crab. Tissue and Cell 4: 137-171.

Halcrow K. 1976. The fine structure of the carapace integument of Daphnia magna Straus (Crustacea, Branchiopodia). Cell Tissue Res. 169: 267-276.

Hayat MA. 1989. Principles and Techniques of Electron Microscopy - Biological Applications. 3rd Edition. Basingstoke and London: Macmillan Press Scientific and Medical, 1-469.

Reynolds ES. 1963. The use of lead citrate at high $\mathrm{pH}$ as an electron opaque stain in electron microscopy. $J$. Cell Biol. 17: 208.

Skinner DM. 1962. The structure and metabolism of a crustacean integumentary tissue during a moult cycle. Biol. Bull. (Woods Hole, Mass.) 123: 635-647.

Skinner DM. 1985. Interacting factors of the crustacean molt cycle. Amer, Zool. 25: 275-284.

Smith JA, Whitfield PJ. 1988. Ultrastructural studies on the early cuticular metamorphosis of adult female Lernaeocera branchialis (L) (Copepoda, Pennellidae). Hydrobiología 1671 168: 607-616.

Stevenson JR. 1968. Metecdysial molt staging and changes in the cuticle of the crayfish Orconectes sanborni (Faxon). Crustaceana 14: 169-177.

Stevenson JR. 1985. Dynamics of the Integument. In: Bliss DE, Mantel LH. eds. The Biology of Crustacea. Volume 9, Integument, Pigments and Hormonal Processes. Orlando: Pub. Academic Press Inc., 1-42.

Receivved: 1 February 1999 